\title{
Advanced Laser Architecture for the Two- Step Laser Tandem Mass Spectrometer
}

Molly E. Fahey, Steven X. Li, Anthony W. Yu, Stephanie A. Getty

NASA Goddard Space Flight Center

Greenbelt, MD

SPIE Defense and Commercial Sensing 2016

Paper 9834-6 
- Motivation

- L2MS Instrument Overview

- L2MS Laser Architecture

- Preliminary Laser Performance

- Future Work

- Conclusions 
- Motivation

- L2MS Instrument Overview

- L2MS Laser Concept

- Preliminary Laser Performance

- Future Work

- Conclusions 
- Contribute to and compliment our understanding of the processes governing the formation, distribution and evolution of primitive materials throughout the solar system

- Future astrobiology missions will focus on small, primitive bodies and the icy moons of the outer planets that may host diverse organic compounds

- These missions require advanced instrument techniques to fully and unambiguously characterize the composition of surface and dust materials

- Targeted missions include flybys, orbiters, landers and rovers to Europa, Trojan, a main belt asteroid, comet, Kupiter belt object (KBO), Titan, Enceladus or Mars 
- Motivation

- L2MS Instrument Overview

- L2MS Laser Architecture

- Preliminary Laser Performance

- Future Work

- Conclusions 
- Combines TOF-MS (time-of-flight mass spectrometry) and LDMS (laser desorption/ ionization mass spectrometry)

- In situ measurements of non-volatile samples to determine both mass assignments and clues to structural information

- Wavelengths are selected based on key vibrational and electronic resonances in the targeted species aligned with the organic diversity and mineralogy expected for future planetary missions of high priority to NASA

- Uses mid-infrared (MIR) and ultra-violet (UV) wavelengths

- $\quad 2.8-2.9 \mu \mathrm{m}$ (TBD) - IR vibrational resonances of hydrated minerals

- $3.4 \mu \mathrm{m}-\mathrm{C}-\mathrm{H}$ vibration resonance of organic species

- $266 \mathrm{~nm}$ - coincides with a short-lived metastable state in many aromatic molecules

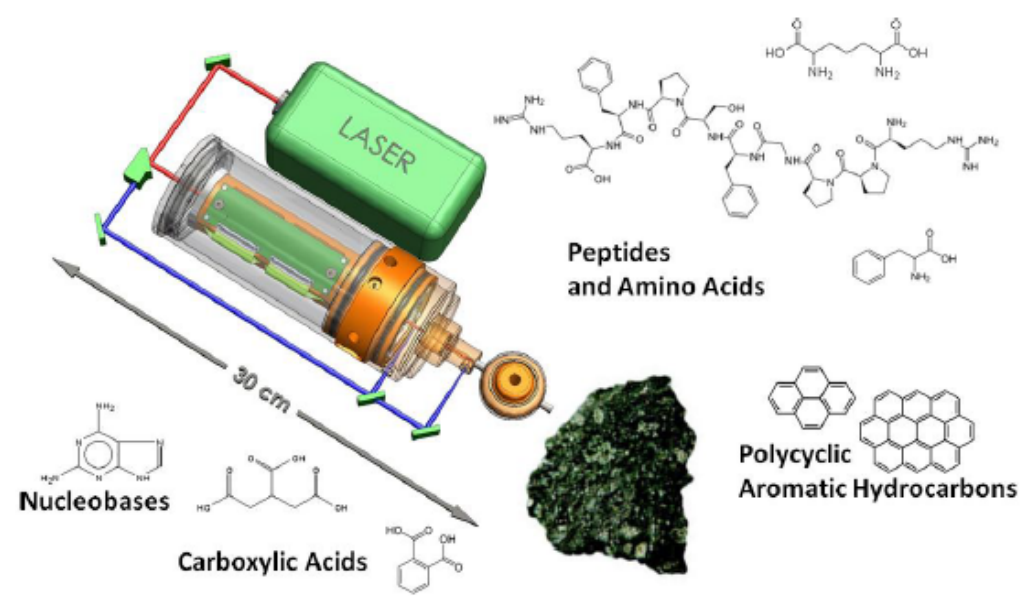


- Molecular desorption is enhanced when the IR laser wavelength matches the molecular vibrational resonance.

- NIST IR transmission measurements:

- Red color indicates regions of decreased transmittance (increased absorption), thus this is the ideal IR wavelength to be used for that compound

- Four different classes of aromatic molecules are

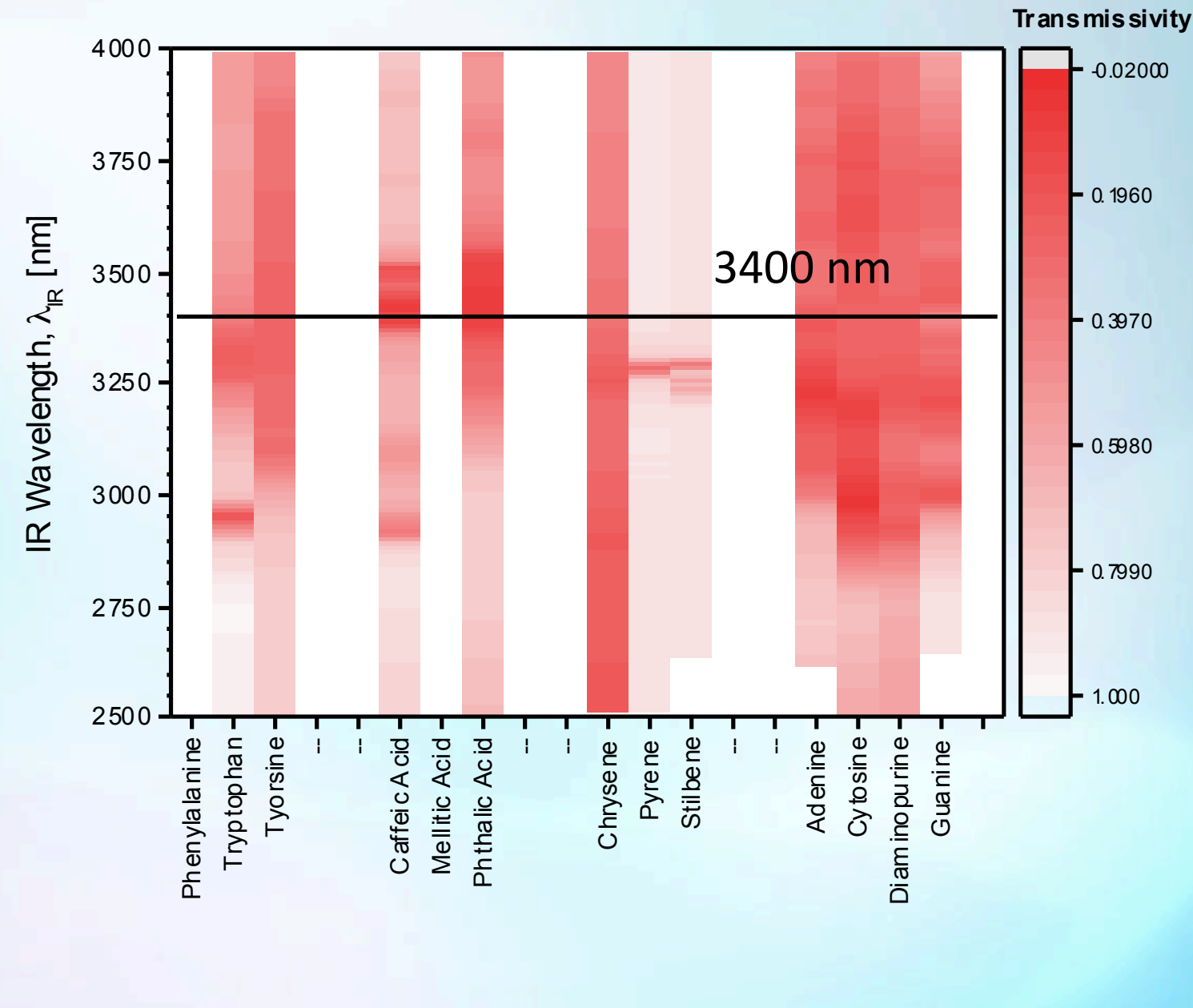
plotted 
- Desorption is enhanced when the IR wavelength matches the mineral absorption resonances

- IR reflectance spectra from USGS (U.S. Geological Survey)or laboratory measurements:

- Red color indicates regions of decreased reflectance (increased absorption), thus an ideal IR wavelength to be used for that mineral.

- Three different classes of minerals are shown

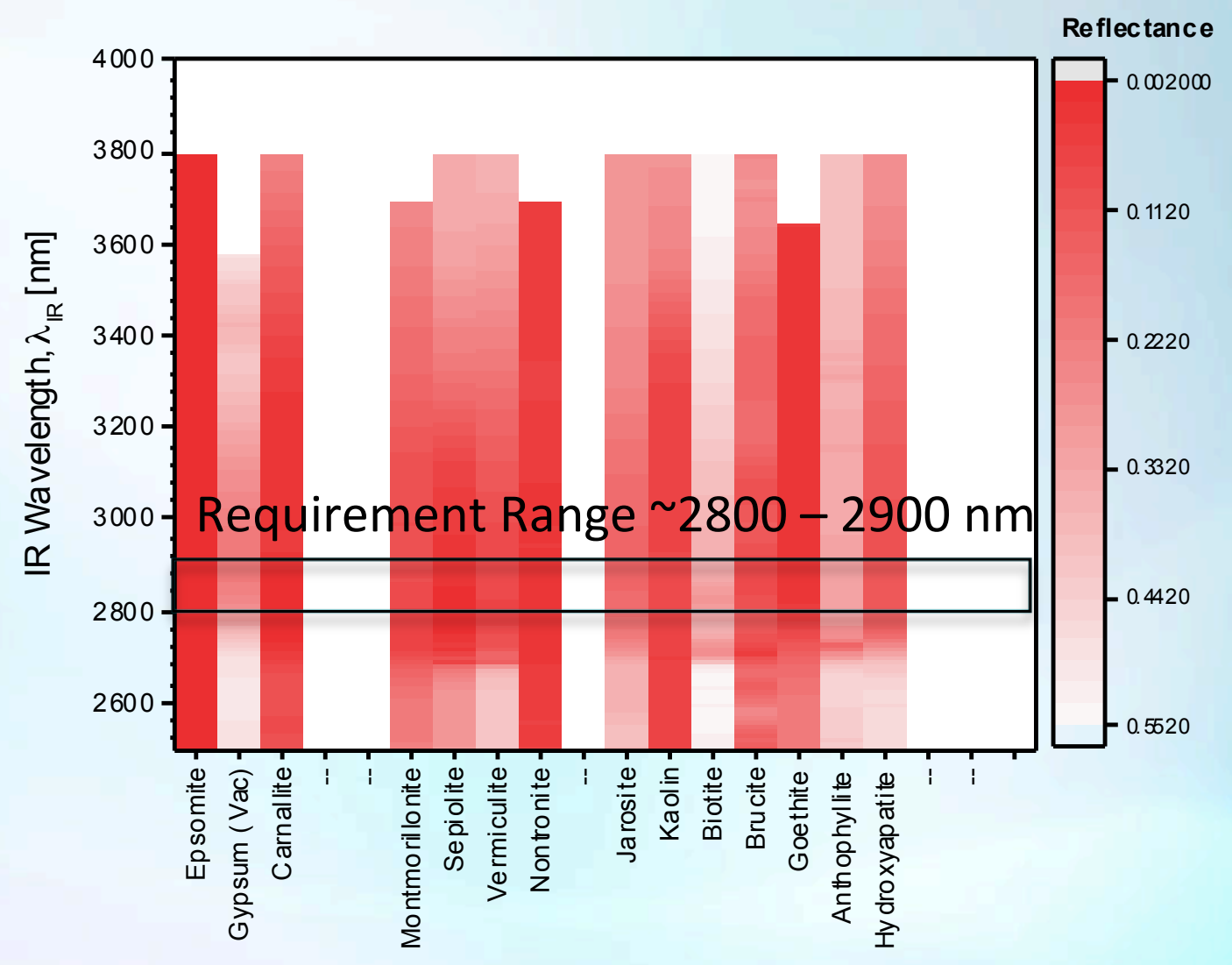


- Two discrete MIR wavelengths ( $2.8 \mu \mathrm{m}$ and $3.4 \mu \mathrm{m})$ and UV wavelength ( $266 \mathrm{~nm}$ ) on the same bench

- Laser design is based on the previously flown Lunar Orbiter Laser Altimeter (LOLA) laser transmitter

- Monolithic intracavity optical parametric oscillators (iOPO) generate the MIR wavelengths

- Frequency quadrupled Nd:YAG generates the UV wavelength

- Typical delays range between 0.3-2 $\mu$ s

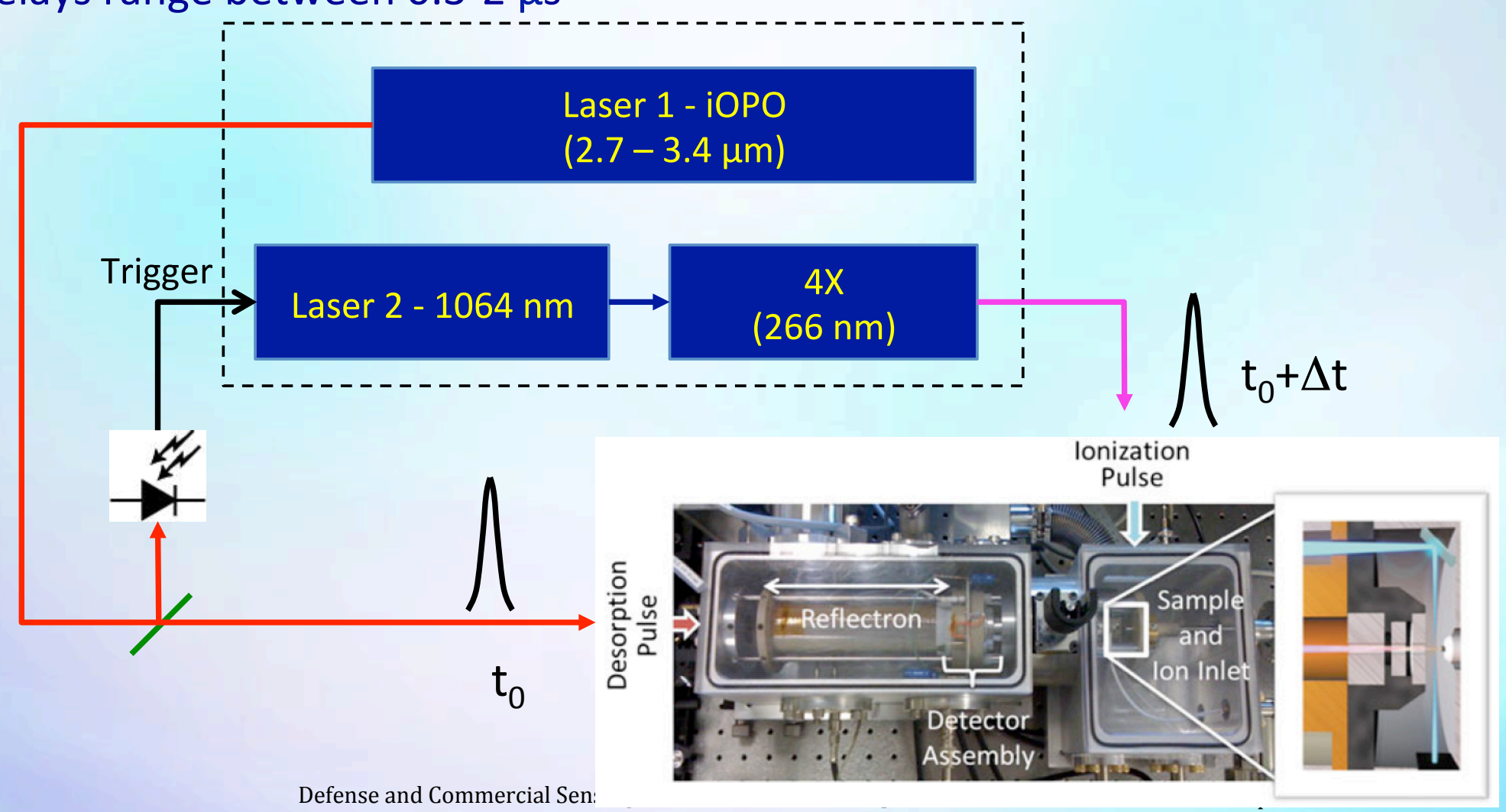



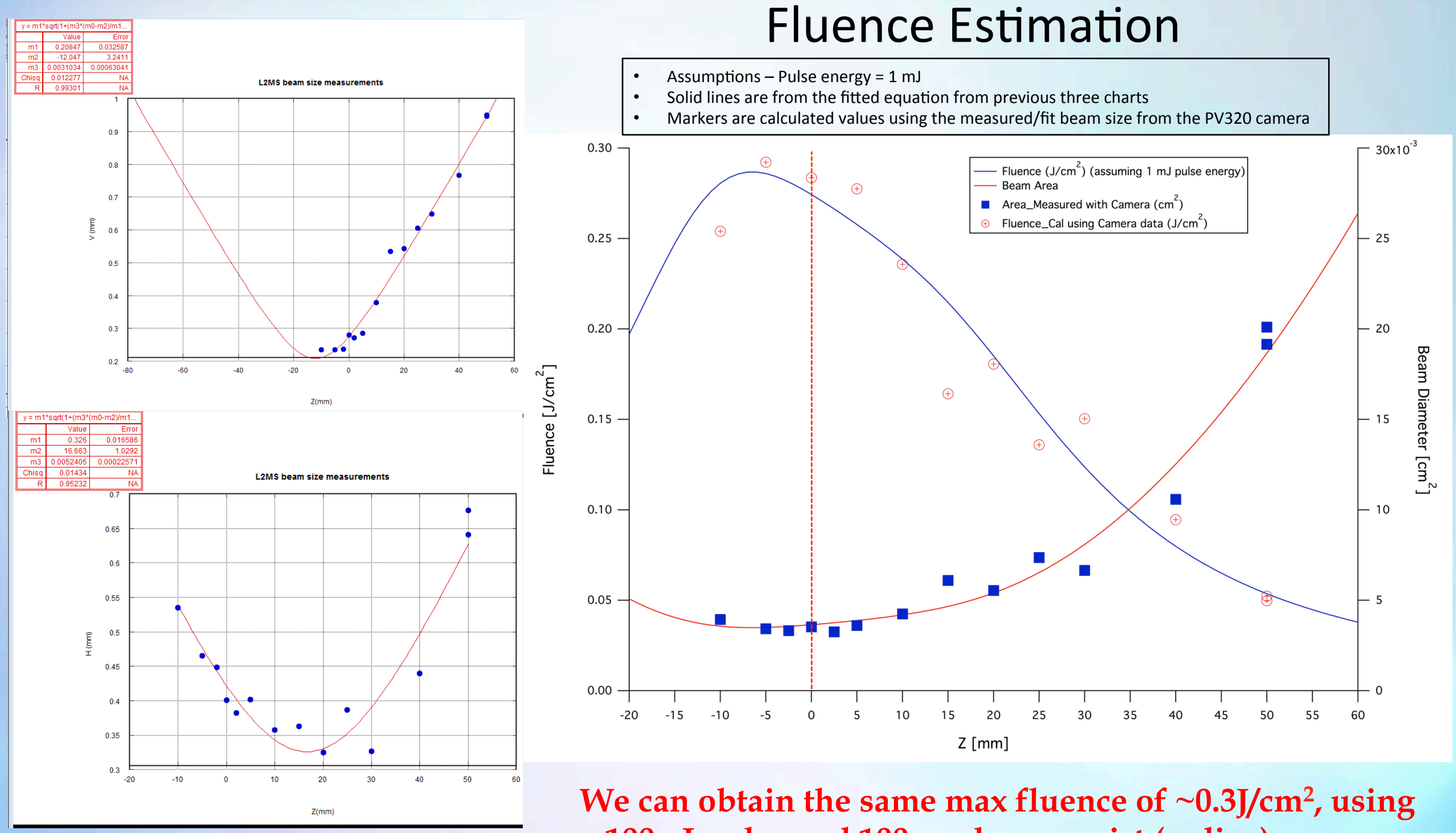

We can obtain the same max fluence of $\sim 0.3 \mathrm{~J} / \mathrm{cm}^{2}$, using a $100 \mu \mathrm{J}$ pulse and $100 \mu \mathrm{m}$ beam waist (radius) 


\begin{tabular}{|l|c|c|}
\hline \multicolumn{1}{|c|}{ Lasers Requirement } & MIR Laser & UV Laser \\
\hline Pulse Repetition Frequency (PRF) & $1-20 \mathrm{~Hz}$ & $1-20 \mathrm{~Hz}$ \\
\hline Wavelength & $2.8 X \mu \mathrm{m}$ and $3.40 \pm 0.05 \mu \mathrm{m}$ & $266 \mathrm{~nm}$ \\
\hline Energy & $\sim 100 \mu \mathrm{J}$ & $\sim 18 \mu \mathrm{J}$ \\
\hline Pulse Width & $<7 \mathrm{~ns}$ & $<7 \mathrm{~ns}$ \\
\hline Peak Power & $\sim 14 \mathrm{~kW}$ & $\sim 2.5 \mathrm{~kW}$ \\
\hline Peak Intensity (assuming $100 \mu \mathrm{m}$ beam diameter) & $180 \mathrm{MW} / \mathrm{cm}^{2}$ & $\sim 30 \mathrm{MW} / \mathrm{cm}^{2}$ \\
\hline Spectral Width & Few GHz & Few GHz \\
\hline Timing & $\mathrm{t}_{0}$ & $\mathrm{t}_{0}+\Delta \mathrm{t} ; \sim 100 \mathrm{~ns}<\Delta \mathrm{t}<\mathrm{few} \mu \mathrm{s}$ \\
\hline Laser Lifetime & 3 year mission at $10 \%$ duty cycle $\sim 64 \mathrm{Mshots} \mathrm{@} 20 \mathrm{~Hz}$ \\
\hline
\end{tabular}


- Motivation

- L2MS Instrument Overview

- L2MS Laser Architecture

- Preliminary Laser Performance

- Future Work

- Conclusions 


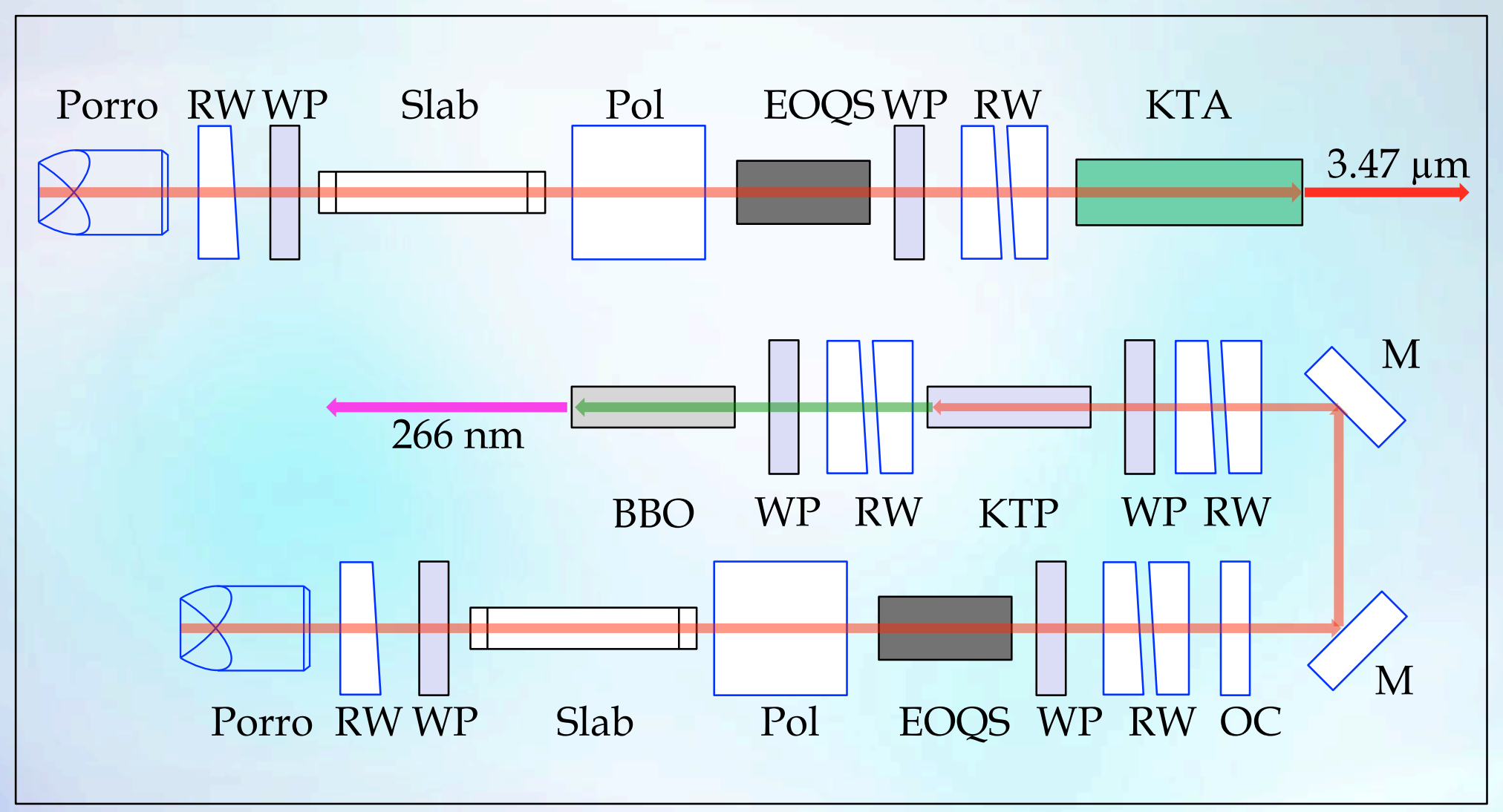

PP - Porro Prism; RW - Risley Wedge; WP - Waveplate; Pol - Polarizer; EOQS - Pockels Cell; M - Mirror; OC - Output Coupler 
- Motivation

- L2MS Instrument Overview

- L2MS Laser Architecture

- Preliminary Laser Performance

- Future Work

- Conclusions 

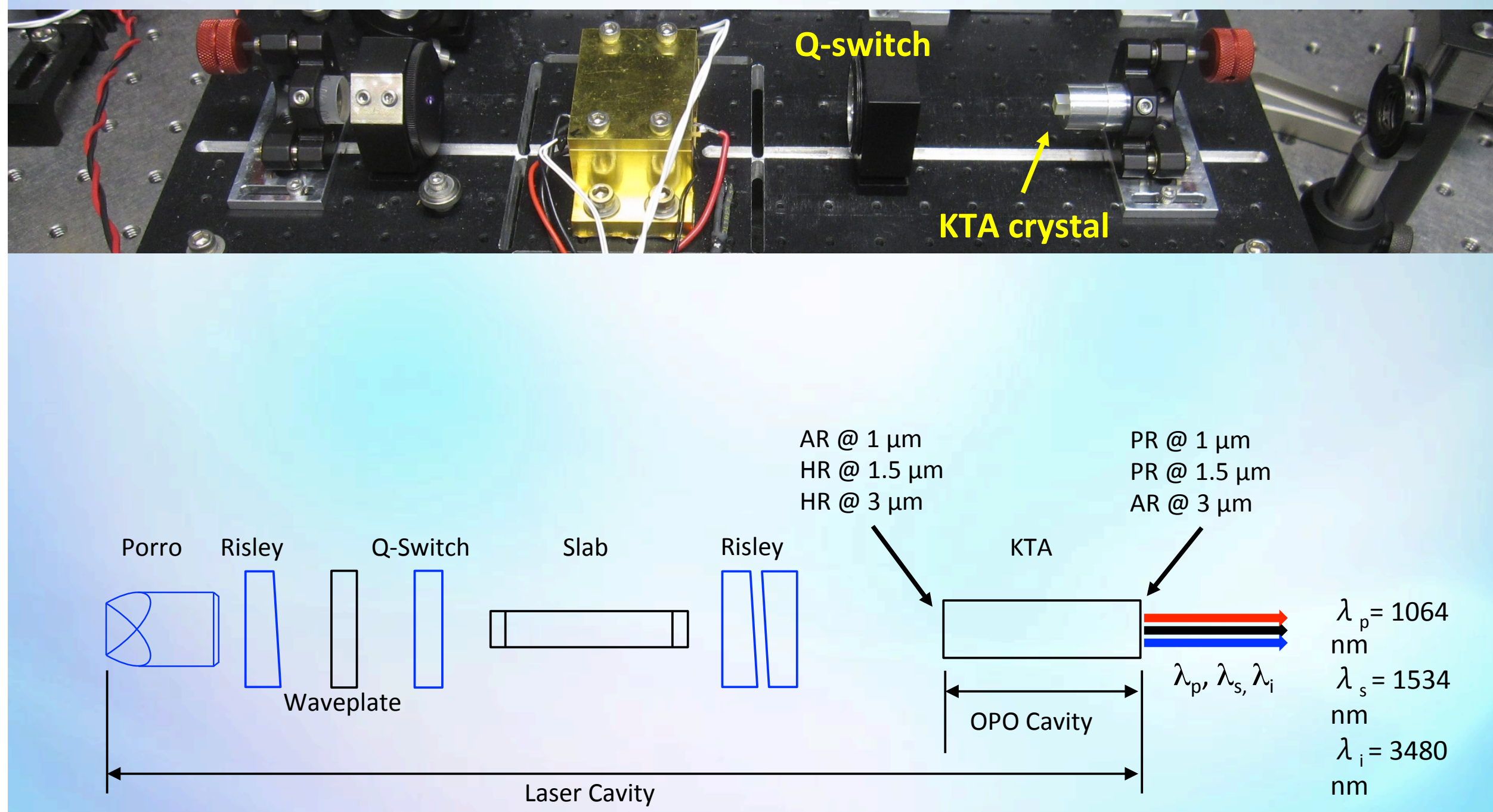


\begin{tabular}{|c|c|}
\hline Parameter & Measurement \\
\hline Wavelength & $3471 \mathrm{~nm}$ \\
\hline
\end{tabular}

Pulse Repetition Frequency $\quad 20 \mathrm{~Hz}$

(PRF)

Average Power

Energy

Pulse Duration

Conversion Efficiency
$4.2 \mathrm{~mW}$

$0.21 \mathrm{~mJ}$

$1.9 \mathrm{~ns}$

$10.5 \%$

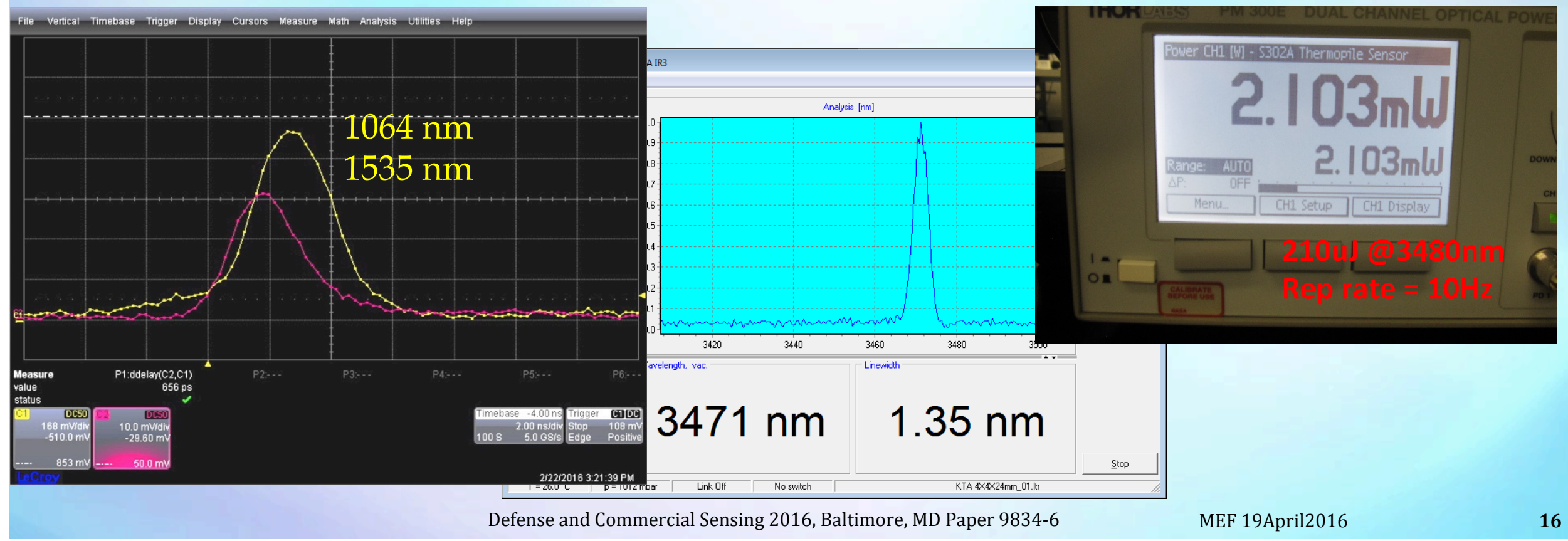




\section{Anst UV Laser Breadboard}

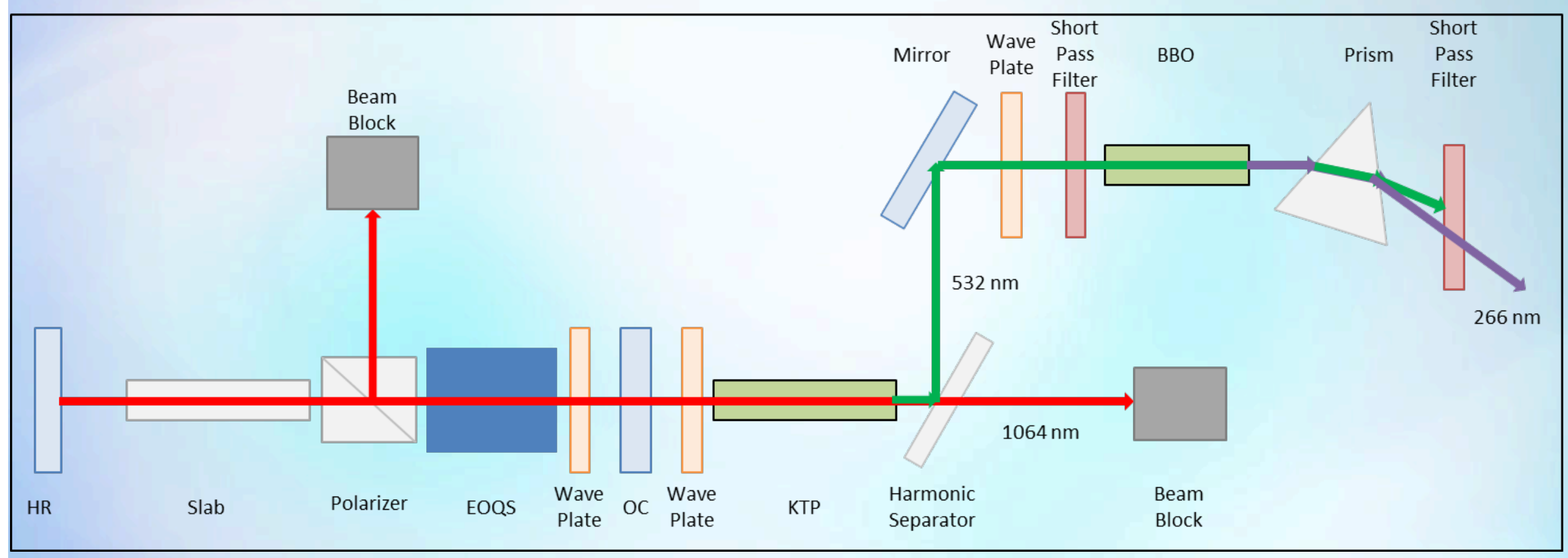




\begin{tabular}{|c|c|}
\hline Parameter & Measurement \\
\hline Wavelength & $1064 \mathrm{~nm}$ \\
\hline Pulse Repetition Frequency (PRF) & $20 \mathrm{~Hz}$ \\
\hline Average Power & $41 \mathrm{~mW}$ \\
\hline Energy & $2.1 \mathrm{~mJ}$ \\
\hline Pulse Duration & $9.8 \mathrm{~ns}$ \\
\hline Peak Power & $210 \mathrm{~kW}$ \\
\hline Divergence (full angle) & $\theta_{\mathrm{x}}=1.53 \mathrm{mrad}$ \\
\hline
\end{tabular}

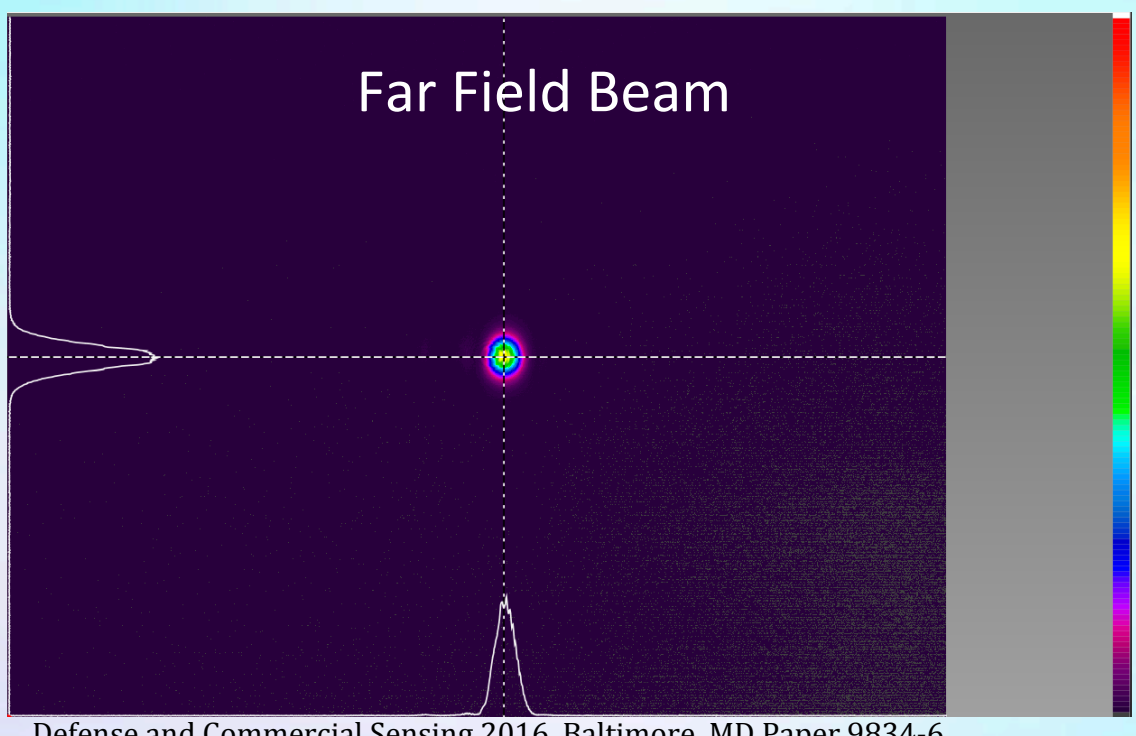


NA3 532 nm Laser Performance

\begin{tabular}{|c|r|}
\hline Parameter & Measu \\
\hline Wavelength & 532 \\
\hline Pulse Repetition Frequency (PRF) & 20 \\
\hline Average Power & $26 \mathrm{~m}$ \\
\hline Energy & 1.3 \\
\hline Pulse Duration & 8.7 \\
\hline Peak Power & 150 \\
\hline Divergence (full angle) & $\theta_{\mathrm{x}}=1.53$ \\
\hline $2^{\text {nd }}$ Harmonic Conversion Efficiency & $\theta_{\mathrm{y}}=1.7$ \\
\hline & \\
\hline & \\
\hline & \\
\hline & \\
\hline
\end{tabular}




\section{Nas 266 nm Laser Performance}

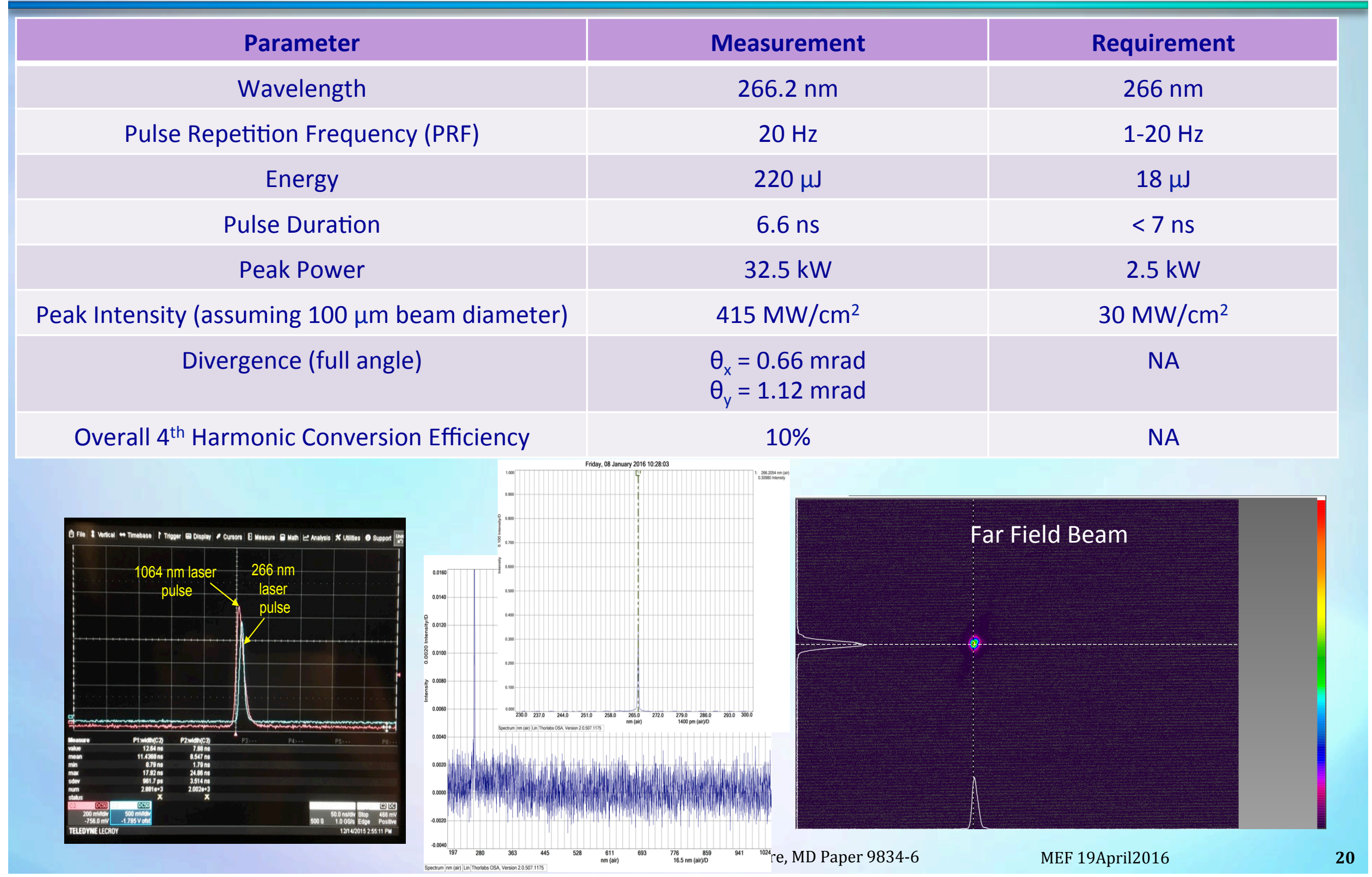


- Motivation

- L2MS Instrument Overview

- L2MS Laser Architecture

- Preliminary Laser Performance

- Future Work

- Conclusions 


\section{Laser 1 - MIR Laser}

- Measure and quantify $3.4 \mu \mathrm{m}$ MIR iOPO breadboard beam quality

- Test $3.4 \mu \mathrm{m}$ breadboard with L2MS laboratory instrument and compare with commercial OPO

- Finalize design for dual wavelength concept

- Continue to work on $2.8 \mu \mathrm{m}$ iOPO breadboard and resolve coating damage issue

Laser 2 - UV Laser

- Replace KTP crystal with critically phase matched LBO crystal for SHG of $532 \mathrm{~nm}$

- Improve mechanical design of LBO crystal housing for better conversion efficiency

- Optimize overall $4^{\text {th }}$ harmonic conversion efficiency

- Test breadboard with L2MS laboratory instrument and compare with commercial UV laser

- Develop mechanical design for mounting optics without using epoxies to avoid contamination issues

Laser Transmitter

- Improve packaging of the laser transmitter for space flight

- Build brass board laser transmitter that will generate both MIR and UV wavelengths on a single laser bench 
- Motivation

- L2MS Instrument Overview

- L2MS Laser Architecture

- Preliminary Laser Performance

- Future Work

- Conclusions 
- We are developing a multi-wavelength laser transmitter for the L2MS Instrument

- A new laser architecture based on the LOLA laser transmitter that generates a single discrete MIR and UV wavelengths has been demonstrated

- The approach provides a straightforward path toward space laser design and deployment

- Preliminary laser breadboard results show compliance with the L2MS instrument requirements 